\title{
Calcite (104) Surface-Electrolyte Structure: A 3D Comparison of Surface X-ray Diffraction and Simulations
}

\author{
Sander J. T. Brugman, Paolo Raiteri, Paolo Accordini, Frank Megens, Julian D. Gale, and Elias Vlieg*
}

Cite This: J. Phys. Chem. C 2020, 124, 18564-18575

Read Online

ABSTRACT: Adsorption and incorporation of ions are known to influence the morphology and growth of calcite. Using surface X-ray diffraction, the interfacial structure of calcite in contact with $\mathrm{CaCO}_{3}, \mathrm{MgCl}_{2}, \mathrm{CaCl}_{2}$, and $\mathrm{BaCl}_{2}$ solutions was determined. All of these conditions yield a comparable interfacial structure, meaning that there is no significant ion adsorption on the terraces under the investigated conditions. This allows, for the first time, a thorough comparison in all three dimensions with state-of-the-art computer simulations, involving molecular dynamics based on both density functional theory (DFT) and two different force field models. Additionally, the simulated structures are used to calculate the corresponding structure factors, which in turn are compared to those obtained from experiment, thereby avoiding the need for fitting or subjective interpretation. In general, there is a good agreement between experiment and the simulations, although there are some small discrepancies in the atomic positions, which lead to an inadequate fit of certain features characteristic of the structure of water at the interface. Of the three simulation methods examined, the DFT results were found to agree best with the experimental structure.

\section{INTRODUCTION}

Several methods have been employed to study the atomic structure of solid-liquid interfaces. Reliable structural data are needed to gain a genuine understanding of the processes occurring at such interfaces. The aim of this investigation is to assess how well we understand the well-studied and relevant calcite (10 $\overline{1} 4)$-aqueous interface in all three dimensions. This is carried out by comparing the independent results from stateof-the-art X-ray diffraction and computer simulations.

The crystal structure accurately provides the bulk atomic positions within an ordered mineral. In contrast, the precise structure on the surface of the mineral is often unknown. A limited number of experimental techniques can be used to investigate the mineral surface structure, especially when the surface is in contact with a liquid. One of the techniques capable of probing such solid-liquid interfaces is surface X-ray diffraction (SXRD) ${ }^{1-3}$ SXRD allows for the determination of the ordered part of the interface, which includes not only the positions of the surface atoms of the crystal but also the partly ordered structure of the liquid at the interface, including hydration shells and adsorbed ions. Recent advances in SXRD, such as the increase in synchrotron brilliance and the use of two-dimensional detectors, allow for rapid acquisition of data sets containing thousands of unique reflections. However, the analysis of these data through fitting has barely changed during the period of such advances. Because of the phase problem, the electron density at the interface cannot be derived directly from the measured intensities. Instead, an initial guess of the structure is made, often based on the bulk crystal structure. During the fitting procedure, the positions, occupancy, and disorder of the atoms are varied to find the structure which best describes the measured data. The disadvantage of this approach is that the structural model has to be constructed manually and features of the interface can remain undiscovered if they are not part of the model. In addition, X-rays are sensitive to the electron density and it can be ambiguous to assign a specific atom to a measured density in combination with the (partial) occupancy of that atom. This is, of course, only a problem if multiple species are present in the system. Finally, SXRD is insensitive to the position of hydrogen atoms. Ideally, these problems can be avoided if the interfacial structure is examined in parallel by computer simulations, such as molecular dynamics (MD) or Monte Carlo methods. Simulations also involve assumptions, but when a consistent picture emerges using two methods, the reliability is high. The combination of diffraction data and simulations has been employed several times, ${ }^{4-9}$ but so far, this never involved directly fitting all three-dimensional atomic coordinates (i.e., height and lateral position) to the structure factors measured with SXRD. This approach can be used to verify the correctness of the simulation structure and does not require any additional interpretation steps.

Another powerful technique to study solid-liquid interfaces is atomic force microscopy (AFM), where technological

Received: May 7, 2020

Revised: July 15,2020

Published: July 28, 2020 
advances have led to a sensitivity that allows probing the liquid ordering at the interface. ${ }^{6,10-12}$ Because of the convolution and interaction with the tip, however, the interpretation of the data can be complex. Reliable (benchmark) structures would therefore be beneficial for the development of AFM as well, including applications in which the local structure imaging of this technique is exploited.

In the present study, calcite is chosen as the solid component of the interface as this is one of the most studied minerals and is often encountered in geological sediments and biomineralization. Aside from the wealth of experimental data available for this system, there has already been extensive use of computer simulation to probe the atomic-level structure of the calcite basal surface. Although earlier studies considered the dry surface ${ }^{13}$ and questions such as whether a $(2 \times 1)$ reconstruction, as reported in surface science experiments, was possible, ${ }^{14,15}$ the focus rapidly shifted to the more relevant interface with aqueous solution. Incorporation of water has been modeled via adsorption of individual molecules or monolayer coverage with optimization based on force fields ${ }^{16}$ and quantum mechanical calculations, ${ }^{17}$ although the majority of studies have employed MD in order to probe the situation in the presence of bulk water. ${ }^{18-20}$ This approach has now made it feasible to study a range of properties spanning the thermodynamics of adsorption of ions and molecules at the calcite terrace $e^{21,22}$ through to the properties of steps and kinks, ${ }^{23,24}$ although accurate simulations of rough surfaces present some challenges because of the lower symmetry and reduced dynamics of water. $^{25}$

The calcite (1014)-water interface was also examined previously using X-ray diffraction, ${ }^{26-30}$ in which mostly calcite in contact with a saturated $\mathrm{CaCO}_{3}$ solution was studied. Besides obtaining experimental data for this well-studied set of conditions, we also investigate three other electrolyte solutions (100 $\mathrm{mM} \mathrm{MgCl}_{2}, 100 \mathrm{mM} \mathrm{CaCl}_{2}$, and $100 \mathrm{mM} \mathrm{BaCl}_{2}$ ), aiming to see the effect of the different ions on the interfacial structure. In all cases, we used $90 \%$ saturated solutions with respect to $\mathrm{CaCO}_{3}$ in order to reduce roughness through moderated etching ${ }^{31}$ and we measured a large number of crystal truncation rods to obtain reliable data sets for comparison with simulations. It has been shown that the presence of $\mathrm{Mg}^{2+}$ ions during crystallization can greatly influence the morphology and growth of $\mathrm{CaCO}_{3}$ crystals. ${ }^{32}$ Furthermore, $\mathrm{Mg}^{2+}$ is more strongly hydrated than the other cations, ${ }^{33}$ which, according to AFM measurements, also leads to a more strongly hydrated calcite interface. ${ }^{34}$ However, in our SXRD experiments, such significant differences were not found when comparing the four sets of conditions. We compared the structural model developed from the SXRD reflections to the structures simulated using MD based on both density functional theory (DFT) and two different force field models. Furthermore, the SXRD signal was calculated for these simulated structures. In general, we find good agreement between the structures, as well as with those reported previously in the literature. A thorough understanding of the structure of the calcite solid-liquid interface is an important step toward simulating more complex systems, such as the interfacial structure close to step edges and defects.

\section{EXPERIMENTAL AND THEORETICAL METHODS}

Experimental Section. Sample Preparation. A calcite single crystal (Iceland spar, MTN Giethoorn) was freshly cleaved using a hammer and directly submerged in a $90 \%$ saturated solution of calcium carbonate, which has a calculated concentration of approximately $0.1 \mathrm{mM}$ for both ions. The $90 \%$ saturated solution of calcium carbonate is prepared by placing calcite crystals in demineralized water for several hours and subsequently adding $10 \%$ demineralized water after the calcite crystals were removed. Based on this procedure, the calcite crystal undergoes a small amount of etching via dissolution, which we found to result in a flat surface, that is, a surface with a roughness factor $\beta$ of $0 .{ }^{35}$ In some experiments, $\mathrm{MgCl}_{2} \cdot 6 \mathrm{H}_{2} \mathrm{O}$ (Sigma-Aldrich, $\geq 99 \%$ pure), $\mathrm{CaCl}_{2} \cdot 2 \mathrm{H}_{2} \mathrm{O}$ (Fluorochem, $\geq 99 \%$ pure), or $\mathrm{BaCl}_{2}$ (Merck, $>99 \%$ pure) was added to this undersaturated solution to make a $100 \mathrm{mM}$ solution of these salts. This gives the following solution compositions: $90 \%$ saturated solution: $0.1 \mathrm{mM} \mathrm{Ca}^{2+}, 0.1 \mathrm{mM}$ total dissolved carbon; $100 \mathrm{mM} \mathrm{Mg}^{2+}: 0.1 \mathrm{mM} \mathrm{Ca}^{2+}, 0.1 \mathrm{mM}$ total dissolved carbon, $100 \mathrm{mM} \mathrm{Mg}^{2+}, 200 \mathrm{mM} \mathrm{Cl}^{-} ; 100 \mathrm{mM}$ $\mathrm{Ca}^{2+}$ : $100.1 \mathrm{mM} \mathrm{Ca}^{2+}, 0.1 \mathrm{mM}$ total dissolved carbon, $200 \mathrm{mM}$ $\mathrm{Cl}^{-}$; and $100 \mathrm{mM} \mathrm{Ba}^{2+}$ : $0.1 \mathrm{mM} \mathrm{Ca}{ }^{2+}, 0.1 \mathrm{mM}$ total dissolved carbon, $100 \mathrm{mM} \mathrm{Ba}^{2+}, 200 \mathrm{mM} \mathrm{Cl}^{-}$. Dissolution of the crystal is expected to saturate the $90 \%$ saturated solution with respect to $\mathrm{CaCO}_{3}$ during the experiment. The $\mathrm{pH}$ of the solution was not adjusted. The amount of released $\mathrm{Ca}^{2+}$ ions by dissolution of the calcite is negligible compared to the concentration of the salt. After an equilibration time of $30 \mathrm{~min}$, the calcite crystal was transferred into the SXRD cell and a few drops of the solution were applied to maintain a stable environment, and then, the crystal was covered by $13 \mu \mathrm{m}$-thick Mylar foil (Lebow Company) to prevent evaporation and was finally mounted on the diffractometer. By gently wiping over the Mylar foil using a tissue, excess liquid was removed. ${ }^{36}$

Surface X-ray Diffraction. SXRD measurements were performed at the I07 beamline of the Diamond Light Source, using a vertical $(2+2)$-type diffractometer and a Pilatus $100 \mathrm{~K}$ area detector. A beam size of $200 \times 20 \mu \mathrm{m}^{2}$ and an X-ray energy of $23 \mathrm{keV}$ were selected. The calcite atomic positions and lattice parameters $(R \overline{3} c, a=b=4.9900 \AA, c=17.061 \AA, \alpha$ $=90.0^{\circ}, \beta=90.0^{\circ}$, and $\gamma=120.0^{\circ}$ in the hexagonal setting) were obtained from Graf. ${ }^{37}$ Anomalous dispersion coefficients at $23 \mathrm{keV}^{38}$ and atomic scattering factors ${ }^{39}$ were used. Four data sets were acquired consisting of the following 19 crystal

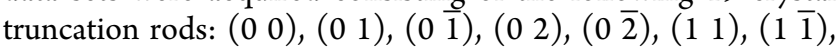

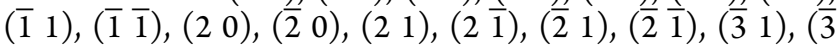
$\overline{1}),(40)$, and $(\overline{4} 0)$. For nonspecular crystal truncation rods, a constant angle of incidence of $1.2^{\circ}$ was used. No changes were observed when some crystal truncation rods were remeasured after a few hours, indicating that the interface is not damaged by the X-ray beam. The agreement factor in the data was determined from symmetry equivalent reflections and lies between 5.5 and $12.2 \%$. Data points close to a Bragg peak were given a larger error of up to $30 \%$ to increase the relative weight of surface-sensitive data points. Using the "ARTS" Matlab script, integrated intensities were converted into structure factors. The acquired data were fitted with a model of the interfacial structure using ROD software. ${ }^{40}$

Simulations. All simulations were performed using $\mathrm{MD}$ based on either force fields or DFT. Obviously, the quality of these simulations will be determined by the choice of the underlying models, especially in the case of the force fields. Over the last decade, the principles of how to construct an accurate force field model for mineral crystallization have been established by paying careful attention not only to structural properties but also the thermodynamics of solvation and solubility. ${ }^{20,41}$ Here, we use a rigid ion model derived based on 
these principles. ${ }^{42}$ However, it is important to recognize that polarizability is important in enhancing the transferability of models between different environments. Although there were earlier attempts to use a shell model representation for water in simulations of aqueous calcium carbonate systems, ${ }^{18}$ this was compromised by the fact that the water model was subsequently shown to freeze at room temperature. ${ }^{41,43}$ Therefore, in this work, we introduce dipolar polarizability alongside static multipoles via a recently developed AMOEBA model for calcium carbonate in contact with water. ${ }^{44}$ The details of the simulation protocols are described below for each specific case.

Rigid Ion Force Field. All MD simulations based on a rigid ion force field were performed with the LAMMPS code ${ }^{45}$ using a 1 fs time step. The simulation cell was created with a 12 layer-thick calcite slab oriented with the [104] direction parallel to the $z$-axis covered with approximately $5 \mathrm{~nm}$ of water. Periodic boundary conditions were applied in all Cartesian directions, and no vacuum spacing was used. The cell was initially equilibrated in the $\mathrm{z}$ direction to ensure that the water had the correct bulk density, and production runs were carried out at a constant volume. The canonical sampling through the velocity rescaling algorithm ${ }^{46}$ with a relaxation time of $0.1 \mathrm{ps}$ was used to keep the temperature at $300 \mathrm{~K}$. The final size of the simulation cell was approximately $50 \times 50 \times 85 \AA^{3}$, and it contained 1440 formula units of $\mathrm{CaCO}_{3}$ and 3914 water molecules. Thirty independent simulations were run using different initial coordinates and velocities. The atomic positions were recorded every $1 \mathrm{ps}$, and a total of 150,000 frames were analyzed using an in-house code to compute the density maps. The molecular interactions were computed using the force field developed in our previous work. ${ }^{42}$ This parametrization has been demonstrated to offer a good description of the thermodynamics of aqueous calcium carbonate systems, including the solubility of calcite.

AMOEBA Force Field. All MD simulations with the polarizable AMOEBA force field were performed with the OpenMM ${ }^{47}$ code and run on GPUs using mixed precision. ${ }^{48,49}$ The same simulation box was used for the AMOEBA calculations as per the rigid ion force field. The MD simulations were run in the NVT ensemble after the cell was equilibrated in the NPT ensemble. The temperature was maintained at $300 \mathrm{~K}$ using a Langevin thermostat with a friction coefficient of 0.1 ps. Three independent simulations were run with a time step of $0.5 \mathrm{fs}$, while the atomic trajectories were recorded every $0.5 \mathrm{ps}$, and a total of 12,000 frames were analyzed.

The molecular interactions were computed using a set of parameters that have been recently developed by some of us to reproduce the solvation free energy of the $\mathrm{Ca}^{2+}$ and $\mathrm{CO}_{3}^{2-}$ ions and the properties of calcite. Details can be found in our previous work. $^{44}$

Density Functional Theory. Ab initio MD (AIMD) simulations of the calcite-water interface were conducted using the Gaussian plane-wave method as implemented in the Quickstep module of CP2K. ${ }^{50}$ All calculations were performed using the BLYP exchange-correlation functional ${ }^{51,52}$ with dispersion corrections using the D3 approach of Grimme and co-workers. ${ }^{53}$ Pseudopotentials of the GTH form ${ }^{54}$ were used with a small core for $\mathrm{Ca}$, while the valence orbitals were expanded using a triple-zeta doubly polarized Gaussian basis set (TZV2P) except for Ca where a double-zeta polarized (DZVP) basis was chosen. An auxiliary basis set of plane waves was used for the electron density with a cutoff of $400 \mathrm{Ry}$. The self-consistent field procedure used the orbital transformation algorithm $^{55}$ with full kinetic preconditioning at the gamma point only. Because of the considerably increased cost of AIMD relative to the force field methods, a relatively small unit cell in the surface plane was used for the simulations with orthorhombic cell parameters of 14.973 by 16.194 by $51.00 \AA^{3}$. This corresponds to six layers of calcite separated by $3 \mathrm{~nm}$ of water with the cell containing 1164 atoms. Hydrogen atoms were replaced by deuterium to improve the integration of the nuclear dynamics, although shorter simulations for hydrogen demonstrated that this had a negligible effect on the interfacial structure to within the statistical fluctuations. MD was performed using a time step $0.5 \mathrm{fs}$, again with the temperature controlled via the canonical sampling through the velocity rescaling algorithm using a relaxation time of 0.01 ps. Simulations were performed at both 300 and $330 \mathrm{~K}$, where the former represents the temperature of interest, while the latter has been proposed for use in AIMD to partially correct for the systematic overstructuring of water because of the choice of the exchange-correlation functional. ${ }^{56}$ Total run lengths of 75 and 125 ps were carried out for the two temperatures.

Calcite Surface Unit Cell. A coordinate transformation was applied to the nonprimitive hexagonal calcite unit cell to obtain a surface unit cell parallel to the basal surface plane. ${ }^{57}$ In this way, the calcite (104) surface plane becomes the (001) plane in the new setting, in which two unit cell vectors lie within the surface plane and the third vector is nearly perpendicular to this. This transformation is given by

$$
\left(\begin{array}{l}
x^{\prime} \\
y^{\prime} \\
z^{\prime}
\end{array}\right)=\left(\begin{array}{lll}
-\frac{3}{8} & 0 & \frac{3}{2} \\
\frac{1}{2} & -1 & 0 \\
\frac{1}{8} & 0 & \frac{1}{2}
\end{array}\right)\left(\begin{array}{l}
x \\
y \\
z
\end{array}\right)
$$

and results in the atomic positions in the new nearly orthorhombic unit cell with $a=8.0960 \AA, b=4.9900 \AA, c=$ 24.2880 $\AA, \alpha=90.0^{\circ}, \beta=90.75^{\circ}$, and $\gamma=90.0^{\circ}$.

Conversion of the Simulation Cell to the Surface Unit Cell. In an SXRD experiment, not only the surface but also a part of the bulk crystal is probed. The simulated structures only contain a finite number of atoms, which is considerably smaller than the volume probed experimentally. ${ }^{4}$ To make the simulated structures compatible with the SXRD data, a few alterations, therefore, have to be made. Specifically, the atomic positions of the simulated interface have to be added on top of the bulk crystal structure of calcite. In addition, force field parameters are typically optimized to reproduce a range of properties, which may include structural, mechanical, and thermodynamic data, while DFT often exhibits systematic errors that depend on the exchange-correlation functional. As a result, simulations will often yield lattice parameters that deviate from experimental values. This would give large discrepancies when compared directly to diffraction data. To overcome these problems, all atomic positions were converted to fractional coordinates, a procedure that essentially normalizes the different unit cells. In the in-plane directions, the simulation has periodic boundary conditions, yielding a precise estimate of the lattice parameters $a$ and $b$. In the perpendicular 

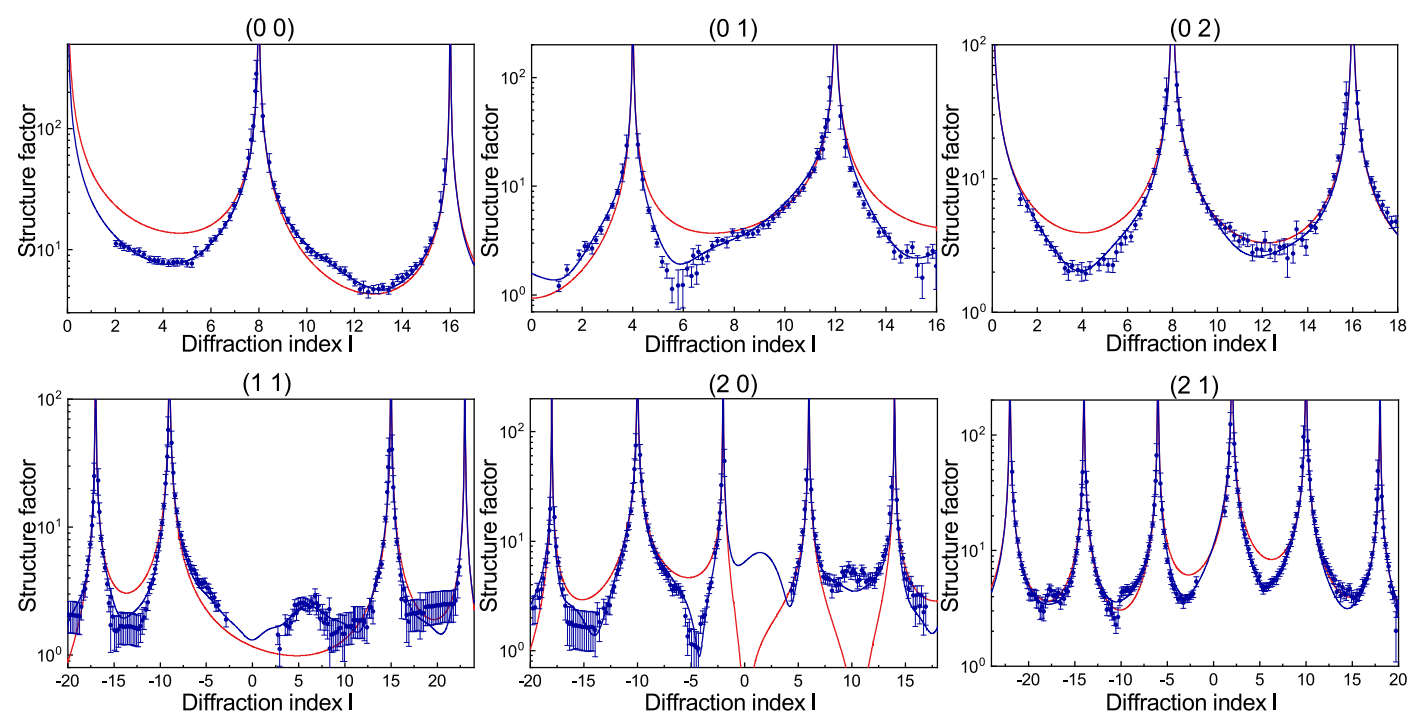

$(3 \overline{1})$
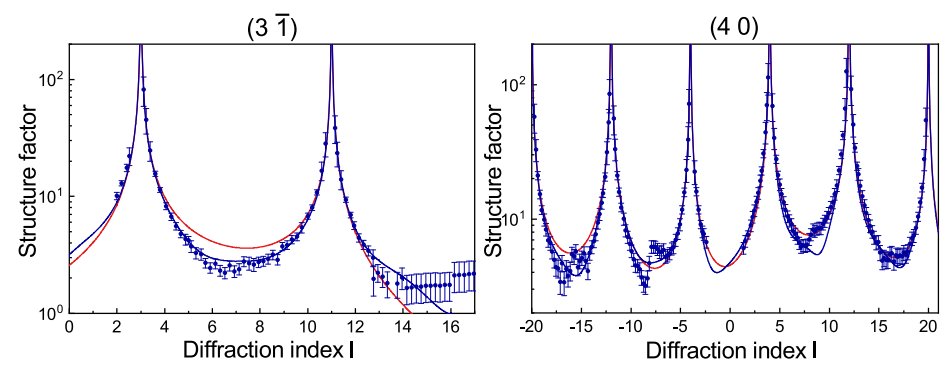

Figure 1. Experimental specular and seven unique nonspecular rods (blue symbols with error bars) for calcite in contact with $100 \mathrm{mM} \mathrm{BaCl}_{2}$. The best model fit, as described in the text, is shown as the blue curve. The red curve shows the rods as calculated for a bulk terminated calcite structure without any liquid molecules.

direction, the simulation only has a limited number of layers and no genuine periodicity for calcite. However, the length of the $c$-axis can be estimated from the distance between the $\mathrm{Ca}^{2+}$ atoms in different layers.

In the literature, often only relaxations in the top two $\mathrm{CaCO}_{3}$ layers were taken into account, as relaxations below these two layers are very small, ${ }^{27,29,58}$ which is also corroborated by our analysis. Therefore, the atomic positions of the top two $\mathrm{CaCO}_{3}$ layers and additional water molecules above the surface, as derived from the simulations, were placed on top of the bulk structure. From the third $\mathrm{CaCO}_{3}$ layer down, the atomic positions were set to the bulk positions, which was carried out for both SXRD and simulated structures. To find the right position to overlay the interface and bulk structure, the $\mathrm{Ca}^{2+}$ atoms in the third layer of the simulated structure were translated to agree with the position of the same atom in the bulk structure.

To derive the atomic positions and occupancies from the simulations, the distribution of the atoms was fitted with a three-dimensional Gaussian distribution per molecule. DebyeWaller (B) parameters were derived from the standard deviation $(\sigma)$ of the Gaussian fit, according to $B=\frac{\pi^{2}(2 \sqrt{2 \ln 2} \sigma)^{2}}{\ln (2)}$. For all (solution) atoms above the crystal, in-plane and out-of-plane Debye-Waller parameters were derived. In the crystal, only isotropic Debye-Waller parameters were used because anisotropy was found to be negligible. Moreover, based on our current analysis, the occupancies of all atoms in the crystal were set to $100 \%$. The derivation of the position, Debye-Waller parameters, and occupancies of the water molecules from the simulations is described in the Supporting Information S1. For a direct comparison with the SXRD model, an isotropic liquid layer, representing the bulk electrolyte solution, was added above the ordered part of the interface. ${ }^{36}$ The simulated structures were used to fit the experimental data without additional fitting parameters, except for the aforementioned isotropic liquid layer and a scale factor.

From our analysis, we find that small changes made during the translation of the simulation cell do not have a large impact on the goodness-of-fit. The distribution of water molecules from the simulations was found to deviate from a Gaussian distribution, and a slightly improved description could often be obtained by fitting the simulated water distribution with two Gaussian distributions, each with a reduced occupancy. This gives a better agreement with the simulated water structure but was found to give only slightly different results in terms of the goodness-of-fit and calculated structure factors. This shows that the conversion from the simulation cell to the surface unit cell is robust and quite independent of the approximations made during the conversion.

\section{RESULTS AND DISCUSSION}

SXRD Experiments. Using SXRD, 12 unique crystal truncation rods were measured for calcite in contact with the four electrolyte solutions mentioned earlier: calcite in contact with a $90 \%$ saturated $\mathrm{CaCO}_{3}$ solution, $100 \mathrm{mM} \mathrm{MgCl}, 100$ $\mathrm{mM} \mathrm{CaCl}_{2}$, and $100 \mathrm{mM} \mathrm{BaCl}_{2}$. The crystal truncation rods of calcite in contact with $100 \mathrm{mM} \mathrm{BaCl}$, shown in Figure 1 , are typical for all experiments. The crystal truncation rods belonging to the other electrolyte conditions can be found in 


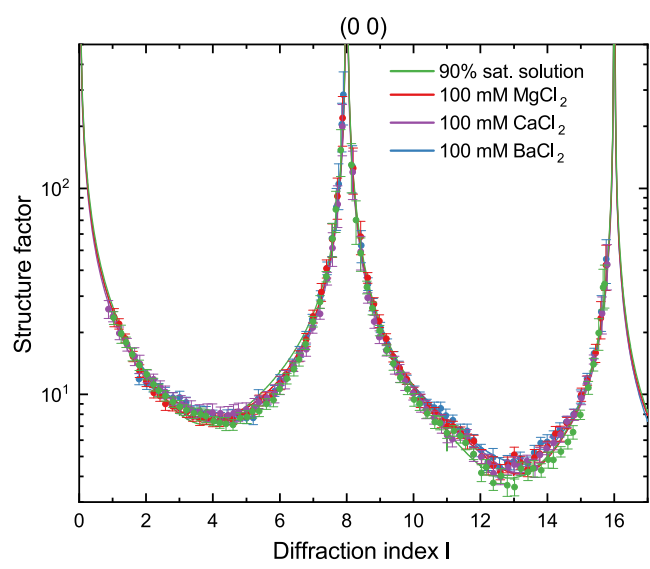

(a)

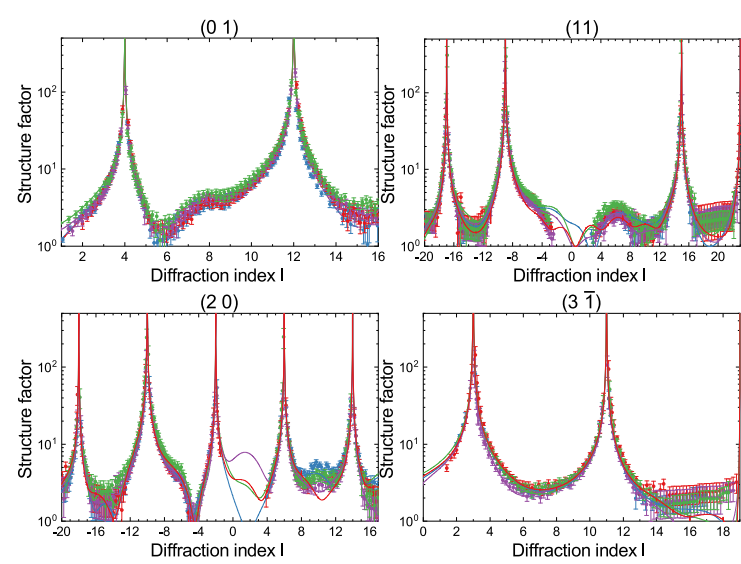

(b)

Figure 2. Experimental (a) specular and (b) a selection of four nonspecular crystal truncation rods for calcite in contact with four electrolyte solutions (symbols with error bars). The best model fits for each set of conditions, as described in the text, are shown as solid curves.

the Supporting Information S2. The red curves in Figure 1 show a calculation for a bulk terminated calcite structure, which clearly does not give a satisfactory fit. The data are fitted using a model that is similar to interface models used previously. ${ }^{27-29}$ In the best fit model (blue curves in Figure 1 ), the bulk atomic positions of calcite were used as initial positions, ${ }^{37}$ followed by selected relaxations: For the calcium atoms in the top two layers, displacements in the $x, y$, and $z$ directions were allowed, while the carbonate ions were considered as a rigid group, allowing fitting of displacements in the $x, y$, and $z$ directions and rotations around the $x, y$, and $z$ axes. The (isotropic) Debye-Waller parameters of the top calcium and carbonate ions were also allowed to vary. The occupancy of the upper calcium carbonate layer was initially varied but was always found to be close to $100 \%$ and was therefore fixed at this value. Above the calcium carbonate layers, two independent water molecules with anisotropic vibrational amplitudes were added. In the structural model, the glide plane symmetry of calcite was retained. This means that two water molecules are present in each water layer per surface unit cell, of which the position of one water molecule defines the position of the other water molecule. Water was modeled as an oxygen atom because SXRD is insensitive to the hydrogen positions. Debye-Waller parameters and occupancies were used as additional fitting parameters for the water molecules. The final component in the model is a liquid layer without in-plane order. This model is found to be the simplest model to give a good fit. A model with only one water molecule instead of two gave a significantly worse fit, whereas adding an extra ordered water layer did not improve the fit significantly.

Any change in the interfacial structure induced by the electrolyte solution should be most noticeable in the specular or (00) crystal truncation rod because this is also sensitive to structural elements without in-plane ordering. This effect is expected to be the most pronounced when looking at the 100 $\mathrm{mM} \mathrm{BaCl}{ }_{2}$ solution because barium is the heaviest element used here and would thus exhibit the strongest X-ray scattering if adsorbed close to the interface. Figure 2 shows the specular and four nonspecular crystal truncation rods for the four different electrolytes. Only minor differences can be observed between the different conditions. Furthermore, we do not see systematic changes in the data when the cation $\left(\mathrm{Mg}^{2+}, \mathrm{Ca}^{2+}\right.$, or $\left.\mathrm{Ba}^{2+}\right)$ is changed. This is in contrast to, for example, sodium nitrate, which is known to induce changes in the top layers of the calcite structure and to disrupt the hydration structure. ${ }^{6}$ Also, the introduction of the $\mathrm{Cl}^{-}$anion does not induce clear changes compared to a solution containing only the $\mathrm{CO}_{3}^{2-}$ anion. This means that for the tested conditions, only a negligible number $(<5 \%)$ of solution cations and anions are adsorbed on the calcite surface or present in the first two water layers at the interface. This can be understood in the view of the low reactivity of the flat calcite surface caused by the strong hydration of the ions in solution relative to the thermodynamic benefit of displacing surface water in order to adsorb on the terrace. ${ }^{6}$ At step edges and kinks, where higher coordination numbers are possible through adsorption, the effect of the ions is expected to be the most visible. ${ }^{6}$ Also, more weakly hydrated ions than the divalent cations used in this study can enter the hydration layers, as was shown for $\mathrm{Rb}^{+}$ions. ${ }^{59}$ Moreover, for our experimental conditions, the incorporation of ions into the crystal structure is not expected because the solution is not supersaturated with respect to calcium carbonate.

Our computer simulations show that indeed $\mathrm{Ca}^{2+}, \mathrm{Mg}^{2+}$, and $\mathrm{Sr}^{2+}$ do not adsorb close to the calcite surface as they only exhibit either a point of inflection or an unstable minimum with a depth that is less than the ambient thermal energy [see the Supporting Information (Figure S12) for more details]. Although $\mathrm{Ba}^{2+}$ has a more pronounced minimum, it is again unstable with respect to the ion in solution and is situated on top of the second water layer. On the other hand, a more significant (local) minimum in the adsorption free energy is observed for $\mathrm{Cl}^{-}$at a height of approximately $2.75 \AA$, where 0 is defined as the bulk height of the topmost $\mathrm{Ca}^{2+}$ layer and corresponds to the chloride ion between the two ordered water layers. However, again a considerable free energy barrier $(\approx 30$ $\mathrm{kJ} / \mathrm{mol}$ ) has to be overcome for adsorption at this site to occur. At the concentrations of the present experiments, only a very small fraction of $\mathrm{Cl}^{-}$is expected to adsorb. An attempt to fit the SXRD data with a model containing this additional $\mathrm{Cl}^{-}$ adsorption site resulted in a worse fit. This means that $\mathrm{Cl}^{-}$has a low occupancy at this site $(<5 \%)$, if present at all.

Because of the small differences in the interfacial structures of the four different conditions, from now on, we will consider the interfacial structure averaged over the four data sets. The differences between the structures give an indication of the accuracy in the SXRD structure determination. In the following sections, we will shift our focus from the different 
electrolyte solutions to the comparison of the experimental data with state-of-the-art simulations.

Experimental Calcite Interfacial Structure. The interfacial structure was determined by fitting the measured crystal truncation rods (Figures 1 and S2) and is shown in Figures 3

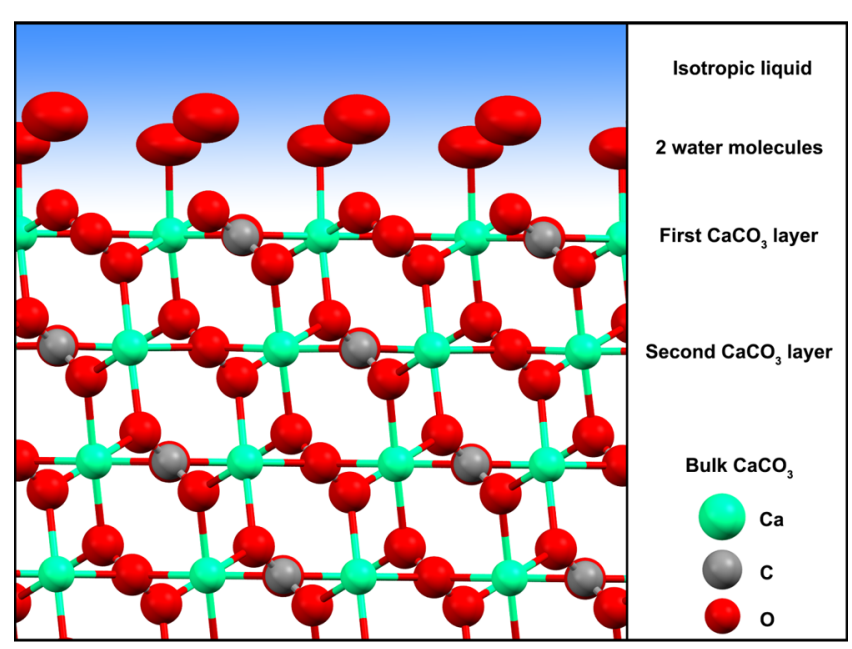

Figure 3. Schematic illustration of the model of the interfacial structure of calcite in contact with an electrolyte solution, viewed along the $b$-axis. The size of the drawn atoms is determined by their (anisotropic) thermal vibration amplitude.

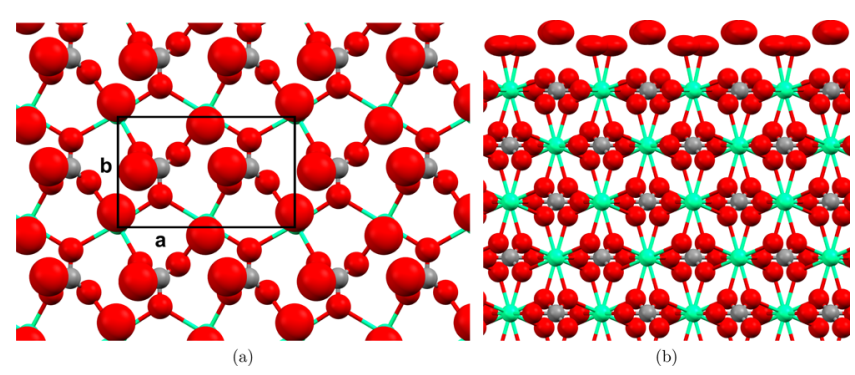

Figure 4. (a) Top view and (b) side view along the $a$-axis of the calcite interfacial structure. The rectangle in (a) indicates the surface unit cell used here. For the color coding of the atoms, we refer to Figure 3.

and 4. Two independent water molecules are found at heights of $2.36 \pm 0.03$ and $3.17 \pm 0.08 \AA$ above the calcite surface, as can be seen from the electron density perpendicular to the surface (i.e., the (00) Fourier component) shown in Figure 5. The inset of this figure displays the (11) Fourier component, which only shows atoms with in-plane order. ${ }^{60}$ The electron density of both water peaks in the (11) Fourier component is similar to the (00) Fourier component, and this indicates that these two water layers are strongly ordered. The next layer, however, is completely disordered. Such an abrupt change in ordering has also been seen for other minerals, such as brushite, ${ }^{9,61}$ quartz, ${ }^{62}$ and rhodochrosite. ${ }^{63}$ The dynamic nature of hydrogen bonding in water apparently results in a more rapid decay of ordering moving away from the surface compared to metals, for example, where layering can exist over many more layers. ${ }^{64-67}$ However, additional layering has also been reported for water. ${ }^{68}$ An AFM study ${ }^{12}$ and some of our simulations discussed later indicate the existence of additional

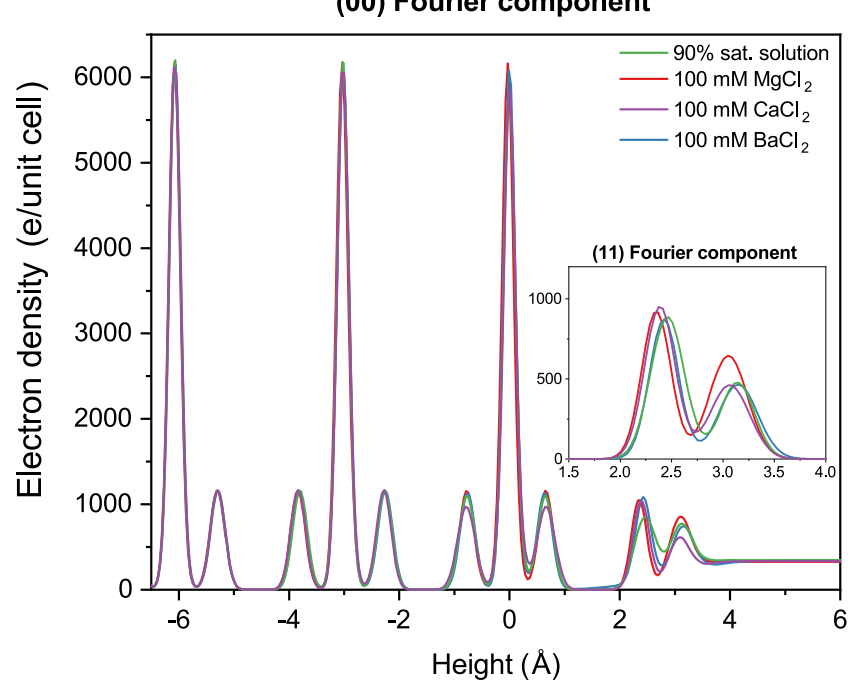

Figure 5. Electron density perpendicular to the surface for calcite in contact with a $90 \%$ saturated $\mathrm{CaCO}_{3}$ solution and $100 \mathrm{mM}$ solutions of $\mathrm{MgCl}_{2}, \mathrm{CaCl}_{2}$, and $\mathrm{BaCl}_{2}$. The inset shows the (11) Fourier component.

hydration layers. In light of these findings, we have investigated if the addition of a third water molecule would improve the fit to the SXRD data. Such an additional water layer does indeed improve the fit slightly, but the water molecule was found at an unreasonable distance from the surface, where such an ordering should not occur. This means that a third ordered water layer does not exist or is too disordered to observe using our data. These findings do not agree with AFM measurements by Araki et al., where the addition of $\mathrm{Mg}^{2+}$ ions leads to two additional detectable water layers. ${ }^{34}$ Potentially, this could be caused by different experimental conditions as the solutions used in the AFM experiments are supersaturated with respect to calcium carbonate, whereas our solutions are undersaturated. Also, differences in sensitivity between the two techniques could play a role.

The water molecule closest to the surface is located directly above calcium, completing the sixfold oxygen coordination of calcium found in the bulk (Figures 3 and 4). This water molecule has an occupancy of $92 \pm 7 \%$ (Table 1), which means that for each surface $\mathrm{Ca}^{2+}$ ion, nearly one water molecule is available. The average $\mathrm{Ca}-\mathrm{O}$ distance is $2.47 \pm$ $0.05 \AA$, which is slightly longer than a bulk $\mathrm{Ca}-\mathrm{O}$ distance of $2.36 \AA$. The second water molecule is located at an average height of $3.17 \pm 0.08 \AA$. This molecule is located at an average distance of $2.58 \pm 0.08 \AA$ from the topmost oxygen atom of the carbonate group and is probably interacting with this oxygen via a hydrogen bond. This distance is longer than the distance between the topmost oxygen atom of the carbonate group and the $\mathrm{Ca}^{2+}$ atom in the layer above (2.36 $\AA$ ). As will be discussed later, the topmost oxygen atom interacts with the $\mathrm{H}$ atom of the second water molecule, which explains the longer distance. This water layer has an occupancy of $71 \pm 5 \%$.

Only small relaxations compared to the bulk structure are found in the two topmost calcium carbonate layers. $\mathrm{Ca}^{2+}$ atoms retain their bulk positions. We find a small rotation of the carbonate group in the top layer of approximately $2^{\circ}$ in the $x$ direction and $3.5^{\circ}$ in the $y$ direction compared to the bulk structure. This leads to a small inward displacement for the topmost oxygen atoms in the calcite structure. One layer 
Table 1. Structural Parameters of the Two Water Layers at the Solid-Liquid Interface of Calcite ${ }^{a}$

\begin{tabular}{|c|c|c|c|c|c|c|}
\hline parameter & SXRD average & bulk extrapolated & rigid ion & AMOEBA & DFT & literature \\
\hline \multicolumn{7}{|c|}{ First Water Layer } \\
\hline height $(\AA)$ & $2.36 \pm 0.03$ & 2.26 & 2.08 & 2.19 & 2.34 & $1.9-2.5$ \\
\hline $\mathrm{Ca}-\mathrm{O}$ distance $(\AA)$ & $2.47 \pm 0.05$ & 2.36 & 2.27 & 2.34 & 2.42 & $2.3-3.0$ \\
\hline occupancy (\%) & $92 \pm 7$ & 100 & 104 & 98 & 102 & \\
\hline \multicolumn{7}{|c|}{ Second Water Layer } \\
\hline height $(\AA)$ & $3.17 \pm 0.08$ & 3.04 & 3.35 & 3.33 & 3.26 & $3.1-3.5$ \\
\hline O-O distance $(\AA)$ & $2.58 \pm 0.08$ & $2.36^{b}$ & 2.58 & 2.63 & 2.60 & \\
\hline occupancy (\%) & $71 \pm 5$ & 100 & 73 & 80 & 75 & \\
\hline
\end{tabular}

${ }^{a}$ The bulk position of the $\mathrm{Ca}^{2+}$ atom of the top layer is defined as 0 in the $z$ direction. ${ }^{b}$ The bulk $\mathrm{Ca}-\mathrm{O}$ distance, as a reference for the $\mathrm{O}-\mathrm{H}_{2} \mathrm{O}$ distance determined here. For more details, see the text.

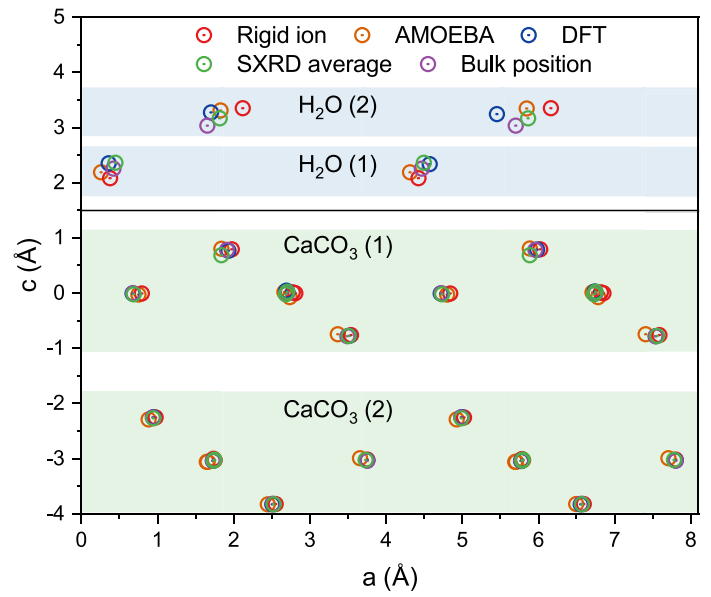

(a)

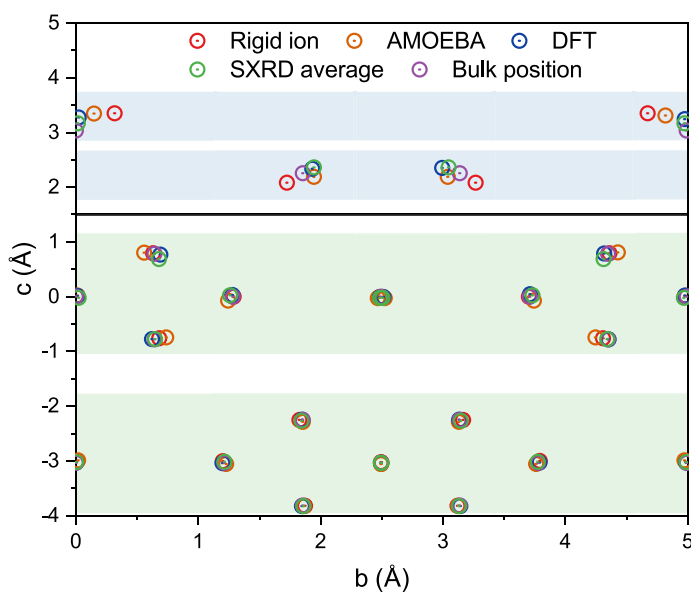

(b)

Figure 6. Atomic positions of the two topmost $\mathrm{CaCO}_{3}$ layers and two water layers in the (a) ac-plane and (b) bc-plane for all methods discussed here. The horizontal black line separates the crystal and the electrolyte solution.

below, these rotations are even closer to zero $\left(<1.5^{\circ}\right)$; thus, the atoms have essentially assumed bulk positions. All structural parameters can be found in the Supporting Information S4.

Our results given in Table 1 compare favorably with the literature, showing that the calcite-water interface is quite well understood. Most studies also find small relaxations in the top calcium carbonate layers with two adsorbed water molecules above these layers. However, when focusing on the details some differences can be seen. In our experiments using the four different solution compositions, the height of the first water layer varies between 2.33 and $2.40 \AA$ above the surface. In the literature, heights of $1.9(1),{ }^{28} 2.07 \pm 0.02,{ }^{4} 2.14 \pm$ $0.02,,^{30} 2.3 \pm 0.1,,^{27} 2.35 \pm 0.10,{ }^{29}$ and $2.50 \pm 0.12 \AA^{26}$ were reported. The distance between this water molecule and the $\mathrm{Ca}^{2+}$ ion $(2.47 \pm 0.05 \AA)$ does agree well with previous experimental and theoretical studies. Heberling et al. reported an average distance of $2.41 \pm 0.1 \AA{ }^{29}$ and Magdans et al. found a distance of $2.4 \AA$, ${ }^{28}$ whereas simulations by de Leeuw and Parker suggested a similar distance of $2.4 \AA$. $^{69}$ On the other hand, Geissbühler et al. found a larger $\mathrm{Ca}-\mathrm{O}$ distance of $2.97 \pm 0.12 \AA$ instead. ${ }^{27}$ Also, the height of the second water molecule, which varies in our experiments between 3.10 and $3.29 \AA$, lies close to literature values $(3.1)^{28} 3.24 \pm 0.12,{ }^{29} 3.37$ $\pm 0.11,{ }^{4} 3.44 \pm 0.11,{ }^{30}$ and $\left.3.45 \pm 0.2 \AA^{27}\right)$. In some of these studies, the occupancies of water were set to $100 \%$. For the first water molecule, this is a good assumption. For the second water layer, this is probably an overestimation as we find an average occupancy of $71 \pm 5 \%$. In summary, our results agree reasonably well with earlier studies. In particular, we find excellent agreement with the most recent SXRD study of the calcite-water interface performed by Heberling et al. ${ }^{29}$

Computational Calcite Interfacial Structure. The calcite interface was simulated using DFT and the rigid ion and AMOEBA force fields. Putting aside specific issues of parametrization or numerical factors, it would be expected that the accuracy of the models should increase going from the nonpolarizable rigid ion force field to AMOEBA, which incorporates induced dipoles and static quadrupole moments. Similarly, DFT should be superior to both force field methods (provided the greater transferability to the interface overcomes any systematic error of the specific functional), although it will be subjected to greater statistical uncertainty because of the necessarily shorter run lengths. In this article, we compare the three simulated structures to the experimental structure in two ways. First, we compare the interfacial structures extracted from all methods. Second, we calculate the crystal truncation rods from the three simulated structures, which are then compared to the experimental crystal truncation rods. The latter allows us to directly compare the experimental data without an intermediate interpretation step (except for the coordinate transformation), that is, the derivation of the interfacial structure from the measured SXRD data.

In all three simulations, the same features as observed in the experimental interfacial structure are present. The simulations show small displacements and rotations in the calcium carbonate layers, and the two water molecules are, at first glance, found close to the expected positions. The atomic positions of the atoms in the first two calcium carbonate layers 
and the two interfacial water layers are shown in Figure 6. All atomic positions and structural parameters can be found in the Supporting Information S5 and S6.

The SXRD measurements show that the positions of the atoms in the second calcium carbonate layer can be approximated by the bulk positions, which are accurately reproduced by both the rigid ion force field and DFT. All methods find rotations of the carbonate group of less than $1^{\circ}$, which is in good agreement with the experiment. AMOEBA fails to accurately describe the first two crystalline layers, which is because the method exhibits a larger error in the $c / a$ ratio for the bulk structure of calcite than the other methods. The rigid ion model also finds inaccurate positions for the interfacial calcite layer, mainly in the $a$ direction. On the other hand, atomic positions in these two layers determined by DFT are in excellent agreement with the SXRD positions and bulk positions, except for the topmost $\mathrm{O}$ atoms. According to SXRD, these atoms undergo a small inward relaxation compared to the bulk position, which is not captured by any of the three simulation techniques. In all simulations, the rotations of the topmost carbonate group are smaller than those in the SXRD structure and therefore do not show the inward relaxation. Regarding the first water layer, the height derived from DFT is in agreement with the experimentally found height, whereas the other simulation techniques find the $\mathrm{O}$ atom too close to the surface (Table 1). This difference in height is worse for the rigid ion force field than for AMOEBA. The discrepancy in this case can be explained by the parametrization of the model against the hydration free energy: In the absence of polarization, it is necessary for the first solvent shell of a $\mathrm{Ca}^{2+}$ ion to be too close in order to capture the thermodynamics correctly. The position of the second water layer is only roughly captured by the simulations, although DFT has the best agreement with SXRD in height. Overall, we can conclude that DFT is significantly better than the two force field techniques, as would be expected, and is capable of reproducing the crystalline layers and giving the closest agreement with the positions of the water layers. The atomic positions of the two crystalline layers of the DFT structure differ, on average, by $0.02 \AA$ from the experimental study, while for the rigid ion and AMOEBA force fields, the difference is $0.05 \AA$ for both. The same holds for the first and second water layers, which differ, on average, by $0.09 \AA$ from the experimental values for DFT, whereas the AMOEBA and rigid ion force fields differ by $0.11 \AA$ and $0.23 \AA$, respectively.

Another way to compare the results is to look at the projected electron density (Figure 7). This shows that the deviations in the height in the crystalline layer are actually very small. The occupancies of all atoms in the crystal are set to $100 \%$; therefore, differences in intensity are only caused by varying heights and Debye-Waller factors. For the water layers, we again see the good agreement of DFT with the experiment, whereas the heights of the two force field simulations differ. The occupancies of the first (98-104\%) and second $(73-80 \%)$ water layers in simulations are close to the experimental values ( $92 \pm 7 \%$ and $71 \pm 5 \%$, respectively), giving rise to peaks in the electron density projection with an area comparable to experiment.

Subsequently, we have used the simulated structures to directly fit the experimental crystal truncation rods. These results are shown in Figure 8 for the (00), (01), (11), and (02) rods, which are the most sensitive to the structure of the interface. The other rods can be found in the Supporting

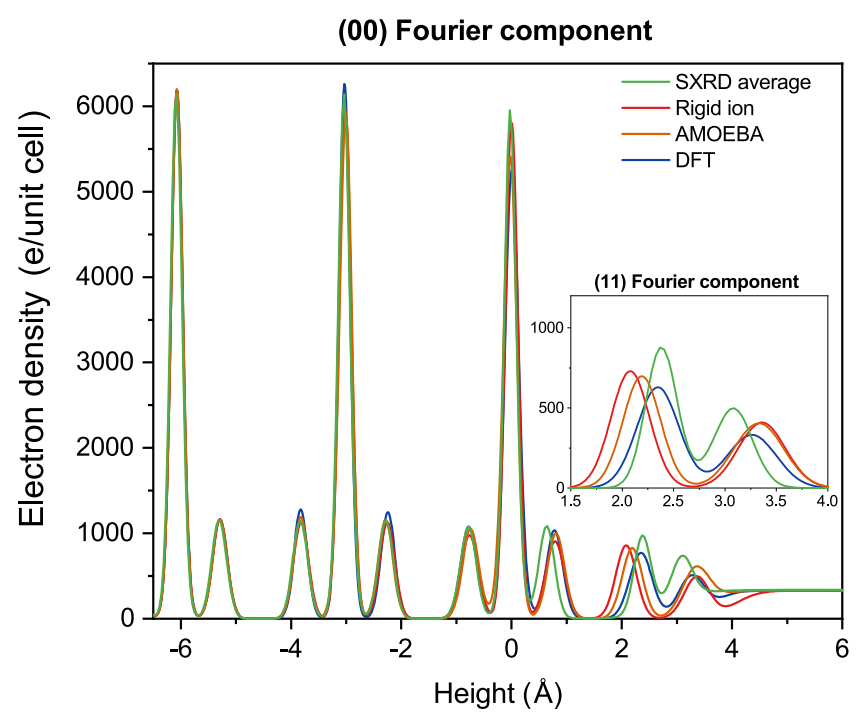

Figure 7. Electron density perpendicular to the surface for calcite in contact with the average of the four sets of experimental conditions shown in comparison to the three simulation results. The inset shows the (11) Fourier component.

Information S7. The DFT structure gives a satisfactory fit to the specular or (00) rod. We noticed earlier, when looking at Figure 7, that the heights in the DFT structure are in good agreement with the SXRD structure. Because the (00) rod is only sensitive to the height and not to the lateral position, a good fit was anticipated. Surprisingly, none of the simulations fully reproduces the "bumps" at $6<l<9$ in the $(01)$ rod and at $3<l<9$ in the (11) rod. These features mainly originate from the ordered water molecules, and they appear to be strongly affected by the positions of the water molecules. By changing the occupancies of the water layers, we find that the feature in the (01) rod is strongly affected by both water molecules, whereas the feature in the (11) rod only depends on the first water layer. Although the DFT structure seems to resemble the experimental structure the most, this is less clear, visually, when judging the fits of the crystal truncation rods. More objectively, the DFT fit has the best goodness-of-fit with an average normalized $\chi^{2}$ value of 4.6 , compared to 6.8 for the rigid ion force field and 8.9 for AMOEBA. All of these values are worse than the value of 2.1 for the model fit, as expected. The good agreement of the simulations partly arises from the correction used for differences in the bulk lattice parameters. Without this correction, the agreement is much worse.

Because of their low X-ray scattering cross section, $\mathrm{H}$ atoms are nearly invisible in SXRD experiments, and therefore, the orientations of water molecules cannot be determined from the experiments. The coupling with simulations allows us to find out the position of the $\mathrm{H}$ atoms (see Figure 9). As expected, the $\mathrm{H}$ atoms of the first water molecule are pointing away from the $\mathrm{Ca}^{2+}$ atom as the $\mathrm{O}$ atom completes its coordination sphere. The $\mathrm{H}$ atoms in the second water layer are pointing toward the topmost $\mathrm{O}$ atom of the carbonate group with a distance of $1.64 \AA$ between them, according to the rigid ion model. Water adsorption thus compensates the broken bonds at the interface. Please note that the different simulations yield somewhat different positions for the $\mathrm{H}$ atoms, but their average positions give a reliable orientation of the water molecules with respect to the calcite surface. 

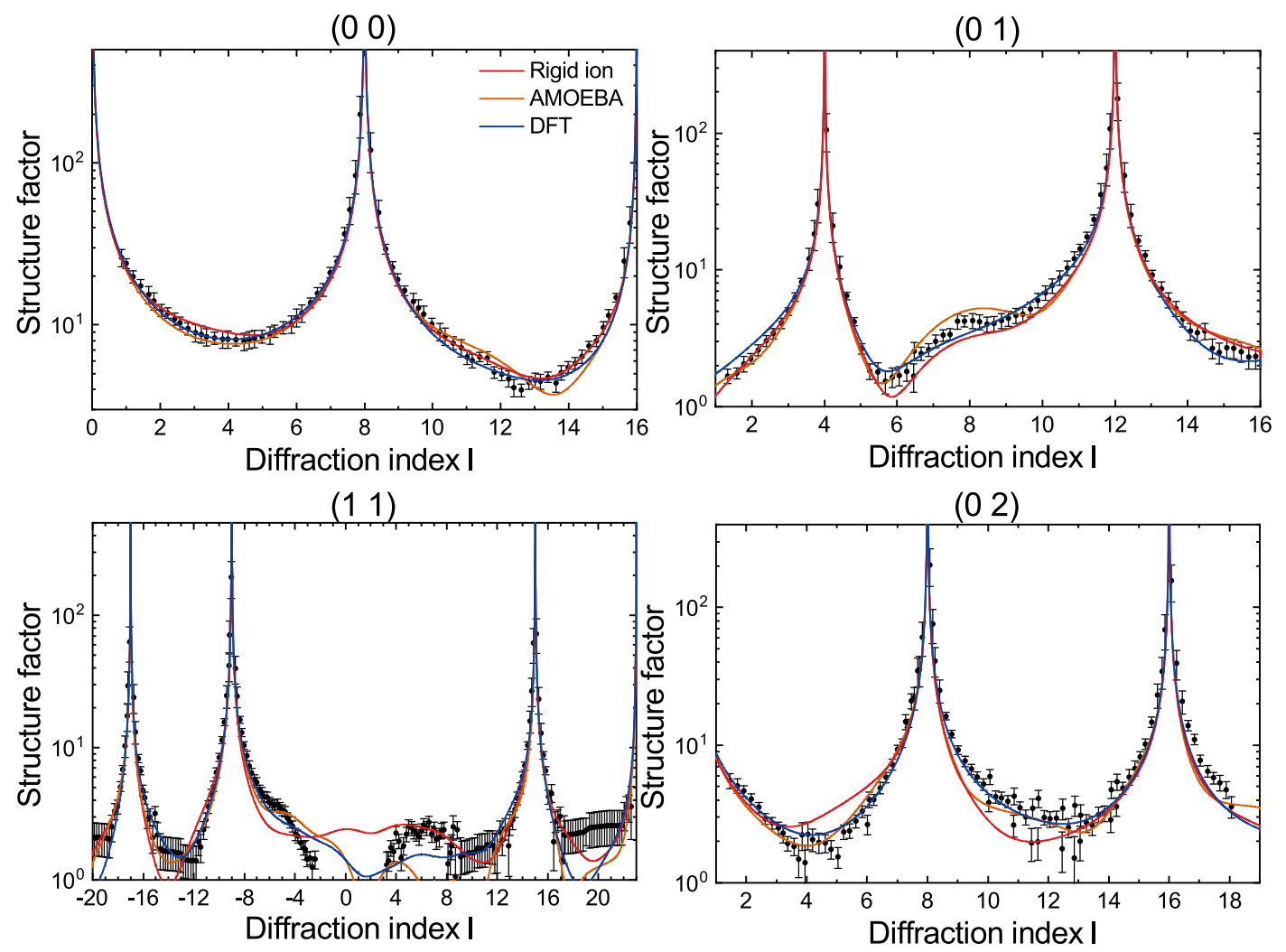

Figure 8. Selection of the measured nonspecular crystal truncation rods for calcite in contact with $100 \mathrm{mM} \mathrm{CaCl}_{2}$, typical for all measured experimental conditions. The fits using the simulated structure are shown as solid lines.

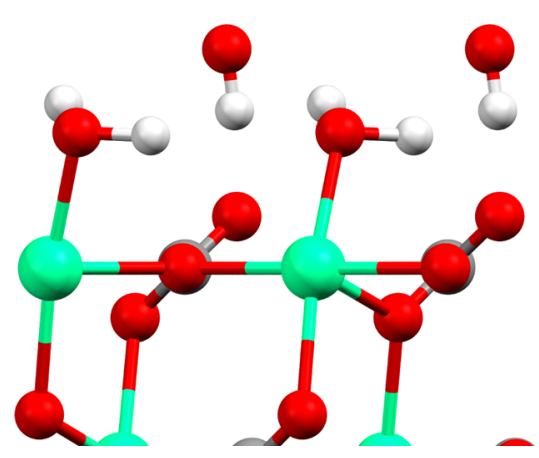

Figure 9. Side view of the calcite interfacial structure, along the $b$-axis, as determined by the rigid ion model. $\mathrm{H}$ atoms are shown in white. For the color coding of the atoms, we refer to Figure 3. The positions of the $\mathrm{H}$ atoms represent the dominant orientation, as derived from the average over a distribution of positions.

Although SXRD provides valuable information on the average structure of the calcite-water interface, one thing that cannot be addressed is whether the ordered water layers are static or dynamic. Here, simulation provides additional data regarding the mean residence time for water molecules on the calcite surface. Although Wolthers et al. ${ }^{70}$ have suggested a mean residence time of only $42 \mathrm{ps}$ at calcite terraces, which implies faster water exchange than for $\mathrm{Ca}^{2+}$ in solution, this is likely to be because vibrational modes were not excluded from the analysis. Analysis of the results for the present rigid ion water model by De La Pierre et $\mathrm{al}^{25}$ yielded a more realistic mean residence time of $2 \mathrm{~ns}$, which represents 1 order of magnitude longer than that for a cation is bulk solution. Here, we have performed the same analysis for the AMOEBA-based model using simulations with a Nose-Hoover thermostat in order to obtain more correct time-correlation data. This results in a mean residence time for water on the calcite surface of the order of $4 \mathrm{~ns}$, which is similar to or greater than that for the rigid ion model, despite the fact that water exchange is faster for $\mathrm{Ca}^{2+}$ in solution with AMOEBA. ${ }^{44}$ Unfortunately, it is not feasible to obtain a comparable value for the DFT simulations because of the run lengths being shorter than the mean residence time. Based on the dynamical information available, it can be ascertained that the absence of adsorbed ions at the calcite terraces under the present conditions is a consequence of the thermodynamics, rather than any kinetic inhibition due to the ordered water layers.

\section{CONCLUSIONS}

Using SXRD, the interfacial structure of calcite (1014) terraces in contact with four different electrolyte solutions was measured. The structures only varied marginally, showing that the presence of $\mathrm{Mg}^{2+}, \mathrm{Ca}^{2+}, \mathrm{Sr}^{2+}, \mathrm{Ba}^{2+}$, and $\mathrm{Cl}^{-}$ions does not significantly affect the structure under the tested conditions. A good fit of the measured SXRD rods is obtained with a model containing the calcite bulk structure with two calcite layers and two water molecules on top, which were free to vary. The final interfacial model is generally in good agreement with previously reported structures.

The experimentally found structure was compared to the structures simulated using DFT and two force field-based methods. For a proper comparison, we use fractional coordinates to avoid issues with differences in lattice constants that arise from simulations. Although there is good agreement on the major features present at the interface, there are still some discrepancies in the atomic positions. This has a large effect on the fits of the experimental data with the simulated 
structures, which turn out to inadequately fit certain characteristic features. Overall, we assess that the DFT structure agrees best with the experimentally found structure. The agreement between SXRD and DFT means that we can state that the structure of the calcite-water interface is known with an accuracy of $0.1 \AA$. This could be used as a benchmark for understanding the liquid features that are found in AFM measurements. A consistent three-dimensional picture from these three important techniques would be an important step in reliably applying these methods to the solid-liquid structure at steps, that is, the location where processes such as growth and reactions take place.

\section{ASSOCIATED CONTENT}

\section{SI Supporting Information}

The Supporting Information is available free of charge at https://pubs.acs.org/doi/10.1021/acs.jpcc.0c04094.

Computational determination of the structural parameters, plots of the simulated atomic density, tables of the simulated and experimental positions of the atoms near the interface, plots of the full X-ray data sets and model fits, and calculation of ion adsorption (PDF)

\section{AUTHOR INFORMATION}

\section{Corresponding Author}

Elias Vlieg - Institute for Molecules and Materials, Radboud University, 6525 AJ Nijmegen, The Netherlands; 이이.org/ 0000-0002-1343-4102; Email: e.vlieg@science.ru.nl

\section{Authors}

Sander J. T. Brugman - Institute for Molecules and Materials, Radboud University, 6525 AJ Nijmegen, The Netherlands

Paolo Raiteri - Curtin Institute for Computation/The Institute for Geoscience Research (TIGeR), School of Molecular and Life Sciences, Curtin University, Perth 6845, Western Australia, Australia; (1) orcid.org/0000-0003-0692-0505

Paolo Accordini - Institute for Molecules and Materials, Radboud University, 6525 AJ Nijmegen, The Netherlands

Frank Megens - Institute for Molecules and Materials, Radboud University, 6525 AJ Nijmegen, The Netherlands

Julian D. Gale - Curtin Institute for Computation/The Institute for Geoscience Research (TIGeR), School of Molecular and Life Sciences, Curtin University, Perth 6845, Western Australia,

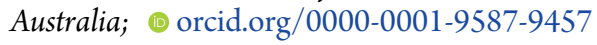

Complete contact information is available at:

https://pubs.acs.org/10.1021/acs.jpcc.0c04094

\section{Notes}

The authors declare no competing financial interest.

\section{ACKNOWLEDGMENTS}

This work is part of the Industrial Partnership Programme Rock-on-a-Chip that is carried out under an agreement between BP Exploration Operating Company Limited and the Netherlands Organisation for Scientific Research (NWO). The experiments were performed on beamline I07 at the Diamond Light Source, Didcot, United Kingdom. We are grateful to Jonathan Rawle for providing assistance in using this beamline. P.R. and J.D.G. thank the Australian Research Council for funding and the Pawsey Supercomputing Centre and National Computational Infrastructure for the provision of computing time.

\section{REFERENCES}

(1) Robinson, I. K.; Tweet, D. J. Surface X-ray diffraction. Rep. Prog. Phys. 1992, 55, 599.

(2) Fenter, P. A. X-ray reflectivity as a probe of mineral-fluid interfaces: A user guide. Rev. Mineral. Geochem. 2002, 49, 149-221.

(3) Vlieg, E. Surface and Interface Science. In Concepts and Methods; Wandelt, K., Ed.; Wiley, 2012; Vol. 1.

(4) Fenter, P.; Kerisit, S.; Raiteri, P.; Gale, J. D. Is the calcite-water interface understood? Direct comparisons of molecular dynamics simulations with specular X-ray reflectivity data. J. Phys. Chem. C 2013, 117, 5028-5042.

(5) Skelton, A. A.; Fenter, P.; Kubicki, J. D.; Wesolowski, D. J.; Cummings, P. T. Simulations of the quartz (1011)/water interface: a comparison of classical force fields, ab initio molecular dynamics, and X-ray reflectivity experiments. J. Phys. Chem. C 2011, 115, 20762088.

(6) Hofmann, S.; Voïtchovsky, K.; Spijker, P.; Schmidt, M.; Stumpf, T. Visualising the molecular alteration of the calcite (104)-water interface by sodium nitrate. Sci. Rep. 2016, 6, 21576.

(7) Bourg, I. C.; Lee, S. S.; Fenter, P.; Tournassat, C. Stern Layer Structure and Energetics at Mica-Water Interfaces. J. Phys. Chem. C 2017, 121, 9402-9412.

(8) Harmon, K. J.; Chen, Y.; Bylaska, E. J.; Catalano, J. G.; Bedzyk, M. J.; Weare, J. H.; Fenter, P. Insights on the Alumina-Water Interface Structure by Direct Comparison of Density Functional Simulations with X-ray Reflectivity. J. Phys. Chem. C 2018, 122, 26934-26944.

(9) Garcia, N.; Raiteri, P.; Vlieg, E.; Gale, J. Water structure, dynamics and ion adsorption at the aqueous $\{010\}$ brushite surface. Minerals 2018, 8, 334

(10) Fukuma, T.; Kobayashi, K.; Matsushige, K.; Yamada, H. True atomic resolution in liquid by frequency-modulation atomic force microscopy. Appl. Phys. Lett. 2005, 87, 034101.

(11) Ricci, M.; Spijker, P.; Stellacci, F.; Molinari, J.-F.; Voïtchovsky, K. Direct visualization of single ions in the Stern layer of calcite. Langmuir 2013, 29, 2207-2216.

(12) Söngen, H.; Reischl, B.; Miyata, K.; Bechstein, R.; Raiteri, P.; Rohl, A. L.; Gale, J. D.; Fukuma, T.; Kühnle, A. Resolving point defects in the hydration structure of calcite (10.4) with threedimensional atomic force microscopy. Phys. Rev. Lett. 2018, 120, 116101.

(13) Kristensen, R.; Stipp, S. L. S.; Refson, K. Modeling steps and kinks on the surface of calcite. J. Chem. Phys. 2004, 121, 8511-8523.

(14) Stipp, S. L.; Hochella, M. F., Jr. Structure and bonding environments at the calcite surface as observed with $\mathrm{X}$-ray photoelectron spectroscopy (XPS) and low energy electron diffraction (LEED). Geochim. Cosmochim. Acta 1991, 55, 1723-1736.

(15) Rohl, A. L.; Wright, K.; Gale, J. D. Evidence from surface phonons for the $(2 \times 1)$ reconstruction of the (104) surface of calcite from computer simulation. Am. Mineral. 2003, 88, 921-925.

(16) Wright, K.; Cygan, R. T.; Slater, B. Structure of the (1014) surfaces of calcite, dolomite and magnesite under wet and dry conditions. Phys. Chem. Chem. Phys. 2001, 3, 839-844.

(17) Lardge, J. S.; Duffy, D. M.; Gillan, M. J.; Watkins, M. Ab initio simulations of the interaction between water and defects on the calcite (1014) surface. J. Phys. Chem. C 2010, 114, 2664-2668.

(18) de Leeuw, N. H.; Parker, S. C.; Harding, J. H. Molecular dynamics simulation of crystal dissolution from calcite steps. Phys. Rev. B: Condens. Matter Mater. Phys. 1999, 60, 13792-13799.

(19) Perry, T. D.; Cygan, R. T.; Mitchell, R. Molecular models of a hydrated calcite mineral surface. Geochim. Cosmochim. Acta 2007, 71, $5876-5887$.

(20) Raiteri, P.; Gale, J. D.; Quigley, D.; Rodger, P. M. Derivation of an Accurate Force-Field for Simulating the Growth of Calcium Carbonate from Aqueous Solution: A New Model for the CalciteWater Interface. J. Phys. Chem. C 2010, 114, 5997-6010.

(21) Kerisit, S.; Parker, S. C. Free energy of adsorption of water and metal ions on the $\{1014\}$ calcite surface. J. Am. Chem. Soc. 2004, 126, 10152-10161. 
(22) Shen, J.-W.; Li, C.; van der Vegt, N. F. A.; Peter, C. Understanding the control of mineralization by polyelectrolyte additives: Simulation of preferential binding to calcite surfaces. $J$. Phys. Chem. C 2013, 117, 6904-6913.

(23) Nada, H. Difference in the conformation and dynamics of aspartic acid on the flat regions, step edges, and kinks of a calcite surface: A molecular dynamics study. J. Phys. Chem. C 2014, 118, 14335-14345.

(24) De La Pierre, M.; Raiteri, P.; Stack, A. G.; Gale, J. D. Uncovering the Atomistic Mechanism for Calcite Step Growth. Angew. Chem., Int. Ed. 2017, 56, 8464-8467.

(25) De La Pierre, M.; Raiteri, P.; Gale, J. D. Structure and dynamics of water at step edges on the calcite (1014) surface. Cryst. Growth Des. 2016, 16, 5907-5914.

(26) Fenter, P.; Geissbühler, P.; DiMasi, E.; Srajer, G.; Sorensen, L. B.; Sturchio, N. C. Surface speciation of calcite observed in situ by high-resolution X-ray reflectivity. Geochim. Cosmochim. Acta 2000, 64, 1221-1228.

(27) Geissbühler, P.; Fenter, P.; DiMasi, E.; Srajer, G.; Sorensen, L. B.; Sturchio, N. C. Three-dimensional structure of the calcite-water interface by surface X-ray scattering. Surf. Sci. 2004, 573, 191-203.

(28) Magdans, U.; Torrelles, X.; Angermund, K.; Gies, H.; Rius, J. Crystalline order of a water/glycine film coadsorbed on the (104) calcite surface. Langmuir 2007, 23, 4999-5004.

(29) Heberling, F.; Trainor, T. P.; Lützenkirchen, J.; Eng, P.; Denecke, M. A.; Bosbach, D. Structure and reactivity of the calcitewater interface. J. Colloid Interface Sci. 2011, 354, 843-857.

(30) Fenter, P.; Sturchio, N. C. Calcite ( $\left.\begin{array}{lll}1 & 0 & 4\end{array}\right)$-water interface structure, revisited. Geochim. Cosmochim. Acta 2012, 97, 58-69.

(31) Townsend, E. R.; Brugman, S. J. T.; Blijlevens, M. A. R.; Smets, M. M. H.; de Poel, W.; van Enckevort, W. J. P.; Meijer, J. A. M.; Vlieg, E. The structure of $\mathrm{PbCl} 2$ on the 100 surface of $\mathrm{NaCl}$ and its consequences for crystal growth. J. Chem. Phys. 2018, 148, 144703.

(32) Davis, K. J.; Dove, P. M.; De Yoreo, J. J. The role of Mg2+ as an impurity in calcite growth. Science 2000, 290, 1134-1137.

(33) Marcus, Y. Thermodynamics of solvation of ions. Part 5.Gibbs free energy of hydration at 298.15 K. J. Chem. Soc., Faraday Trans. 1991, 2995-2999.

(34) Araki, Y.; Tsukamoto, K.; Takagi, R.; Miyashita, T.; Oyabu, N.; Kobayashi, K.; Yamada, H. Direct observation of the influence of additives on calcite hydration by frequency modulation atomic force microscopy. Cryst. Growth Des. 2014, 14, 6254-6260.

(35) Robinson, I. K. Crystal truncation rods and surface roughness. Phys. Rev. B: Condens. Matter Mater. Phys. 1986, 33, 3830.

(36) Brugman, S. J. T.; Townsend, E. R.; Smets, M. M. H.; Accordini, P.; Vlieg, E. Concentration-dependent adsorption of CsI at the muscovite-electrolyte interface. Langmuir 2018, 34, 3821.

(37) Graf, D. L. Crystallographic tables for the rhombohedral carbonates. Am. Mineral. 1961, 46, 1283-1316.

(38) Cromer, D. T.; Liberman, D. A. Anomalous dispersion calculations near to and on the long-wavelength side of an absorption edge. Acta Crystallogr. 1981, 37, 267-268.

(39) Henke, B. L.; Gullikson, E. M.; Davis, J. C. X-ray interactions: photoabsorption, scattering, transmission, and reflection at $\mathrm{E}=50$ 30,000 eV, Z= 1-92. At. Data Nucl. Data Tables 1993, 54, 181-342.

(40) Vlieg, E. ROD: a program for surface X-ray crystallography. J. Appl. Crystallogr. 2000, 33, 401-405.

(41) Raiteri, P.; Demichelis, R.; Gale, J. D. Development of Accurate Force Fields for the Simulation of Biomineralization. Methods Enzymol. 2013, 532, 3-23.

(42) Raiteri, P.; Demichelis, R.; Gale, J. D. Thermodynamically Consistent Force Field for Molecular Dynamics Simulations of Alkaline-Earth Carbonates and Their Aqueous Speciation. J. Phys. Chem. C 2015, 119, 24447-24458.

(43) Van Maaren, P. J.; Van Der Spoel, D. Molecular dynamics simulations of water with novel shell-model potentials. J. Phys. Chem. B 2001, 105, 2618-2626.

(44) Raiteri, P.; Schuitemaker, A.; Gale, J. D. Ion pairing and multiple ion binding in calcium carbonate solutions based on a polarizable AMOEBA force field and ab initio molecular dynamics. J. Phys. Chem. B 2020, 124, 3568-3582.

(45) Plimpton, S. Fast parallel algorithms for short-range molecular dynamics. J. Comput. Phys. 1995, 117, 1-19.

(46) Bussi, G.; Donadio, D.; Parrinello, M. Canonical sampling through velocity rescaling. J. Chem. Phys. 2007, 126, 014101.

(47) Eastman, P.; Swails, J.; Chodera, J. D.; McGibbon, R. T.; Zhao, Y.; Beauchamp, K. A.; Wang, L.-P.; Simmonett, A. C.; Harrigan, M. P.; Stern, C. D.; et al. OpenMM 7: Rapid development of high performance algorithms for molecular dynamics. PLoS Comput. Biol. 2017, 13, No. e1005659.

(48) Friedrichs, M. S.; Eastman, P.; Vaidyanathan, V.; Houston, M.; Legrand, S.; Beberg, A. L.; Ensign, D. L.; Bruns, C. M.; Pande, V. S. Accelerating molecular dynamic simulation on graphics processing units. J. Comput. Chem. 2009, 30, 864-872.

(49) Eastman, P.; Pande, V. S. Efficient nonbonded interactions for molecular dynamics on a graphics processing unit. J. Comput. Chem. 2010, 30, 1268.

(50) Hutter, J.; Iannuzzi, M.; Schiffmann, F.; VandeVondele, J. CP2K: Atomistic simulations of condensed matter systems. Wiley Interdiscip. Rev.: Comput. Mol. Sci. 2014, 4, 15-25.

(51) Becke, A. D. Density-functional thermochemistry. I. The effect of the exchange-only gradient correction. J. Chem. Phys. 1992, 96, $2155-2160$

(52) Lee, C.; Yang, W.; Parr, R. G. Development of the ColleSalvetti correlation-energy formula into a functional of the electron density. Phys. Rev. B: Condens. Matter Mater. Phys. 1988, 37, 785-789.

(53) Grimme, S.; Antony, J.; Ehrlich, S.; Krieg, H. A consistent and accurate $\mathrm{ab}$ initio parametrization of density functional dispersion correction (DFT-D) for the 94 elements H-Pu. J. Chem. Phys. 2010, 132,154104

(54) Goedecker, S.; Teter, M.; Hutter, J. Separable dual-space Gaussian pseudopotentials. Phys. Rev. B: Condens. Matter Mater. Phys. 1996, 54, 1703-1710.

(55) VandeVondele, J.; Hutter, J. An efficient orbital transformation method for electronic structure calculations. J. Chem. Phys. 2003, 118, 4365-4369.

(56) Bankura, A.; Karmakar, A.; Carnevale, V.; Chandra, A.; Klein, M. L. Structure, dynamics, and spectral diffusion of water from firstprinciples molecular dynamics. J. Phys. Chem. C 2014, 118, 29401.

(57) Trainor, T. P.; Eng, P. J.; Robinson, I. K. Calculation of crystal truncation rod structure factors for arbitrary rational surface terminations. J. Appl. Crystallogr. 2002, 35, 696-701.

(58) Magdans, U.; Gies, H.; Torrelles, X.; Rius, J. Investigation of the $\{104\}$ surface of calcite under dry and humid atmospheric conditions with grazing incidence X-ray diffraction (GIXRD). Eur. J. Mineral. 2006, 18, 83-92.

(59) Heberling, F.; Eng, P.; Denecke, M. A.; Lützenkirchen, J.; Geckeis, H. Electrolyte layering at the calcite (104)-water interface indicated by $\mathrm{Rb}+$-and $\mathrm{Se}(\mathrm{VI}) \mathrm{K}$-edge resonant interface diffraction. Phys. Chem. Chem. Phys. 2014, 16, 12782-12792.

(60) Reedijk, M. F.; Arsic, J.; Hollander, F. F. A.; De Vries, S. A.; Vlieg, E. Liquid order at the interface of KDP crystals with water: Evidence for icelike layers. Phys. Rev. Lett. 2003, 90, 066103.

(61) Arsic, J.; Kaminski, D.; Poodt, P.; Vlieg, E. Liquid ordering at the brushite-\{010\}-water interface. Phys. Rev. B: Condens. Matter Mater. Phys. 2004, 69, 245406.

(62) Schlegel, M. L.; Nagy, K. L.; Fenter, P.; Sturchio, N. C. Structures of quartz (100)-and (101)-water interfaces determined by $\mathrm{x}$-ray reflectivity and atomic force microscopy of natural growth surfaces. Geochim. Cosmochim. Acta 2002, 66, 3037-3054.

(63) Jun, Y.-S.; Ghose, S. K.; Trainor, T. P.; Eng, P. J.; Martin, S. T. Structure of the hydrated (10-14) surface of rhodochrosite (MnCO3). Environ. Sci. Technol. 2007, 41, 3918-3925.

(64) Huisman, W. J.; Peters, J. F.; Zwanenburg, M. J.; de Vries, S. A.; Derry, T. E.; Abernathy, D.; van der Veen, J. F. Layering of a liquid metal in contact with a hard wall. Nature 1997, 390, 379.

(65) Algra, R. E.; Vonk, V.; Wermeille, D.; Szweryn, W. J.; Verheijen, M. A.; van Enckevort, W. J.; Bode, A. A.; Noorduin, W. L.; Tancini, 
E.; de Jong, A. E.; et al. Formation of wurtzite InP nanowires explained by liquid-ordering. Nano Lett. 2011, 11, 44-48.

(66) Vonk, V.; Cremers, M.; de Jong, A.; Pintea, S.; Vlieg, E. Atomic layering and misfit-induced densification at the $\mathrm{Si}(111) / \mathrm{In}$ solidliquid interface. Surf. Sci. 2014, 621, 69-76.

(67) de Jong, A.; Vonk, V.; Bockowski, M.; Grzegory, I.; Honkimäki, V.; Vlieg, E. Complex geometric structure of a simple solid-liquid interface: $\mathrm{GaN}(0001)$-Ga. Phys. Rev. Lett. 2020, 124, 086101.

(68) Toney, M. F.; Howard, J. N.; Richer, J.; Borges, G. L.; Gordon, J. G.; Melroy, O. R.; Wiesler, D. G.; Yee, D.; Sorensen, L. B. Voltagedependent ordering of water molecules at an electrode-electrolyte interface. Nature 1994, 368, 444.

(69) de Leeuw, N. H.; Parker, S. C. Surface structure and morphology of calcium carbonate polymorphs calcite, aragonite, and vaterite: an atomistic approach. J. Phys. Chem. B 1998, 102, 29142922.

(70) Wolthers, M.; Di Tommaso, D.; Du, Z.; de Leeuw, N. H. Variations in calcite growth kinetics with surface topography: molecular dynamics simulations and process-based growth kinetcs modelling. CrystEngComm 2013, 15, 5506-5514. 